\title{
ON THE EIGENTHEORY OF OPERATORS WHICH EXHIBIT A SLOW VARIATION*
}

\author{
BY \\ LAWRENCE SIROVICH AND B. W. KNIGHT \\ Rockefeller University and Brown University
}

\begin{abstract}
A class of linear operators which exhibit slow variation is considered. If the kernel of the operator is $K\left(x-y, \frac{1}{2} \varepsilon(x+y)\right)$, $\varepsilon$ the parameter of slowness, then its Wigner transform is defined to be $\tilde{K}(p, q)=\int K(u, q) \exp (-i u p) d u$. The eigenvalues of such operators are shown to follow an area rule: if the curve $\lambda_{n}=\tilde{K}(p, q)$ contains the area $\mathscr{A}(\lambda)=(2 n+1) \pi \varepsilon$ then $\lambda_{n}$ is an eigenvalue. Forms for the corresponding eigenfunctions are also obtained. Classical WKB theory is shown to be a special case and other examples are given.
\end{abstract}

1. Introduction. The present investigation arises from a problem in neural information processing. For background we briefly state that problem. If we consider the visual processing of a spatial pattern of illumination $e(\mathbf{x})$, then response $r(\mathbf{x})$ as measured at some level of the visual pathway can be represented in a reasonable linear approximation by an integral operator

$$
r(\mathbf{x})=\int K\{\mathbf{x}, \mathbf{y}\} e(\mathbf{y}) d \mathbf{y} .
$$

Data imply the further approximation of reciprocity between cause and effect: $K(\mathbf{x}, \mathbf{y}\}=$ $K\{\mathbf{y}, \mathbf{x}\}$ whence the kernel $K$ is symmetric (and Hermitean a fortiori, as it is real). A standard approximation regards the kernel as translationally invariant, $K\{\mathbf{x}, \mathbf{y}\}=$ $K(\mathbf{x}-\mathbf{y})$, in which case the relation takes on the form of a two-dimensional convolution $r=K * e$. In this form $K$ is referred to as the point spread function [1] and its Fourier transform $\tilde{K}(p)$ as the spatial modulation transfer function [2]. The latter is a quantity measurable in the laboratory, and measures the sensitivity of the visual system to sinusoidal patterns. Thus the locus of the maximum $\tilde{K}(\mathbf{p})$ on the p plane indicates those sinusoidal stimuli which yield maximum sensitivity of the visual system. The distinguished role of sinusoidal stimuli arises from their ability to replicate themselves in the response and thereby to yield a simple "coefficient of replication," or eigenvalue, which furnishes a direct measures of the retina's sensitivity to each sinusoid. This distinguished role of sinusoids is manifestly a consequence of the approximation of translational invariance.

Translational invariance is known to be correct only locally $[3,4,5,6]$. However,

* Received March 12, 1980. This work was supported in part by grants from the National Science Foundation (MC 577h08598) and from the U.S. Eye Institute (EY 188, EY 1428, EY 1472). 
regardless of any question of translational invariance, it follows fairly generally from the theory of Hermitean operators that there should be some set of stimuli $\psi(\mathbf{x})$ which are distinguished by the self-replicating property

$$
\int K\{\mathbf{x}, \mathbf{y}\} \psi(\mathbf{y}) d \mathbf{y}=\lambda \psi(\mathbf{x})
$$

where as before the eigenvalue $\lambda$ furnishes a simple numerical measure of the system's sensitivity to the distinguished stimulus $\psi(\mathbf{x})$. Under general conditions the set of eigenfunctions $\psi$ should be complete in the sense that an arbitrary stimulus may be expressed as a superposition of them. In consequence the functions $\psi$ and their corresponding eigenvalues $\lambda$ furnish a full characterization of the way the visual pathway processes an arbitrary stationary stimulus.

Both insight and technical progress on the problem posed may be achieved by first analyzing a one-dimensional caricacture of the two-dimensional problem discussed so far. In this spirit we drop vector notation in what follows.

Aside from the boundary of an eye, which is an example of a sharp inhomogeneity $[7,8]$, departures from homogeneity are found to be relatively gentle. To treat this latter case we first observe that with no loss of generality one may write

$$
K\{x, y\}=K\left(x-y, \frac{1}{2}(x+y)\right)
$$

(the coefficient $\frac{1}{2}$ is convenient for purposes of normalization); and for the case of slow departures from homogeneity we introduce a formal small parameter $\varepsilon$ :

$$
K\{x, y\}=K\left(x-y, \frac{\varepsilon}{2}(x+y)\right) .
$$

Here $\varepsilon$ plays its usual strategic role: since it is the coefficient of an argument which already dictates only gentle functional dependence, results which hold in accurate approximation when $\varepsilon$ is small still hold when $\varepsilon$ is set to unity.

In keeping with our goal of treating problems in neural processing we consider the spectral problem

$$
\int_{-\infty}^{\infty} K\left(x-y, \frac{\varepsilon}{2}(x+y)\right) \psi(y ; \varepsilon) d y=\lambda \psi(x ; \varepsilon) .
$$

The maximum eigenvalue will then correspond to the maximal sensitivity and the corresponding eigenfunction to the most sensitive stimulus pattern. Our treatment of this problem leads to methods valid for fairly general linear operator problems. To underline this we immediately widen the scope of our deliberations by a simple reversal in viewpoint.

We reinitiate our discussion by first considering real functions $\tilde{K}(p, q)$ and then their corresponding transforms

$$
K(u, q)=\frac{1}{2 \pi} \int \tilde{K}(p, q) \exp (i u p) d p .
$$

Then for

$$
u=x-y, \quad q=\frac{\varepsilon}{2}(x+y)
$$


$K$ is a Hermitean kernel and leads to operator problems of the form (1). Depending on the nature of $\widetilde{K}$, the operator $K\{x, y\}$, (2), generated in this manner can be integral, differential or more generally pseudo-differential. The inverse transform $\widetilde{K}(p, q)$ first appeared in a paper by Wigner [9] and we therefore refer to it as the Wigner transform. It has also been used by Groenewald [10], Moyal [11] and more recently by Bruer [12] in alternate descriptions of quantum mechanics.

The relation between $p$ and $q$,

$$
\tilde{K}(p, q)=\lambda,
$$

$\lambda$ constant, generates contour lines in the $(p, q)$-plane which we refer to as $\lambda$-curves. The main result which is shown later states that (1) has the eigenvalue $\lambda_{n}$ when the $\lambda$-curve

$$
\tilde{K}(p, q)=\lambda_{n},
$$

the contour line upon which the value of $\tilde{K}$ is the $n$th eigenvalue $\lambda_{n}$, encloses the area

$$
\mathscr{A}\left(\lambda_{n}\right) \sim(2 n+1) \pi \varepsilon .
$$

As indicated, this result is asymptotic in the slowness parameter $\varepsilon$. We will refer to (5) and (6) and the included statement as the area rule.

2. Perturbation analysis. The manner in which a naive perturbation analysis breaks down often indicates the path a more sophisticated approach must take. In this spirit, a naive approach to solving the eigenvalue problem (1) follows from Taylor expansion of the problem in powers of $\varepsilon$. Thus if we write

$$
\begin{aligned}
\psi(x, \varepsilon)=\psi_{0}(x)+\varepsilon \psi_{1}(x)+\cdots, \\
K\left(x-y, \frac{\varepsilon}{2}(x+y)\right)=K_{0}(x-y)+\frac{\varepsilon}{2}(x+y) K_{1}(x-y)+\cdots,
\end{aligned}
$$

and introduce these into (1) we obtain at lowest order $\left(\varepsilon^{0}\right)$

$$
\int K_{0}(x-y) \psi_{0}(y) d y=\lambda \psi_{0}(x)
$$

for which

$$
\psi_{0}(x)=A \exp (i p x)
$$

solves with arbitrary constants $A$ and $p$, and yields the purported eigenvalue

$$
\lambda=\int_{-\infty}^{\infty} K_{0}(x) \exp [-i p x] d x \equiv \tilde{K}_{0}(p) .
$$

If this procedure is followed to the next order we find that $\psi_{1}$ contains a contribution of $O\left(x \psi_{0}\right)$ which invalidates the procedure when $\varepsilon x=O(1)$. This in turn suggests the remedy that both $A$ and $p$ in (7) be taken as functions of $\varepsilon x$ and that $K$ be given a more careful development.

We now adopt a somewhat different but equivalent approach. At the risk of confusion with (3) we write

$$
q=\varepsilon x,
$$


but as will be seen below this is a consistent notation. Next we expand the eigenfunction $\psi$ as follows:

$$
\psi=\exp \left[\frac{i}{\varepsilon} \phi(q)\right\rfloor \cdot \sum_{n=0} \varepsilon^{n} A_{n}(q),
$$

which is equivalent to the approach followed in the WKB procedure [13]. In addition, we employ a more careful expansion of the kernel

$$
K\left(x-y, \frac{\varepsilon}{2}(x+y)\right)=K_{0}(x-y, q)-\frac{\varepsilon}{2}(x-y) K_{q}(x-y, q)+\frac{\varepsilon^{2}}{8}(x-y)^{2} K_{q q}(x-y, q) .
$$

Finally in the integrand we encounter, for example, $\phi(\varepsilon y)$ which has the expansion

$$
\phi(\varepsilon y)=\phi(q)-\varepsilon(x-y) \phi^{\prime}(q)+\frac{\varepsilon^{2}}{2}(x-y)^{2} \phi^{\prime \prime}(q)+\cdots
$$

and similarly for $A_{n}(\varepsilon y)$. If these are substituted into (1), then at the lowest order we find

$$
\phi=\int^{q} p(s) d s
$$

where $p(q)$ is defined through the Wigner transform, as the solution $p$, for fixed $q$, of the equation

$$
\tilde{K}(p, q)=\int_{-\infty}^{\infty} \exp [-i p u] K(u, q) d u=\lambda .
$$

As mentioned in the introduction, (12) defines a $\lambda$-curve in the $(p, q)$-plane. (Recall $(3)$ that $u=x-y$.) We take up the question of the spectrum later and for the present regard $\lambda$ as a known constant.

If we proceed to the next order we obtain from (1)

$$
\begin{aligned}
\int_{-\infty}^{\infty} \exp [-i u p] K(u, q)\left\{u A_{0}^{\prime}(q)-\frac{i}{2} u^{2} p^{\prime} A_{0}\right\} d u & \\
& +\frac{A_{0}}{2} \int_{-\infty}^{\infty} u K_{q}(u, q) \exp [-i u p] d u=0 .
\end{aligned}
$$

To reduce this further, observe that

$$
\begin{aligned}
\frac{i}{2} \frac{\partial}{\partial q} \tilde{K}_{p}(p, q) & =\frac{1}{2} \frac{\partial}{\partial q} \int_{-\infty}^{\infty} u K(u, q) \exp [-i u p] d u \\
& =\frac{1}{2} \int_{-\infty}^{\infty} u K_{q}(u, q) \exp [-i u p]-d u \frac{i}{2} \int_{-\infty}^{\infty} K(u, q) u^{2} p^{\prime} \exp [-i u p] d u,
\end{aligned}
$$

so that (13) may be rewritten as

or

$$
A_{0}^{\prime}(q) \tilde{K}_{p}+\frac{A_{0}}{2} \frac{\partial}{\partial q} \tilde{K}_{p}=0
$$

$$
\frac{\partial}{\partial q} A_{0} \sqrt{ } \tilde{K}_{p}=0
$$




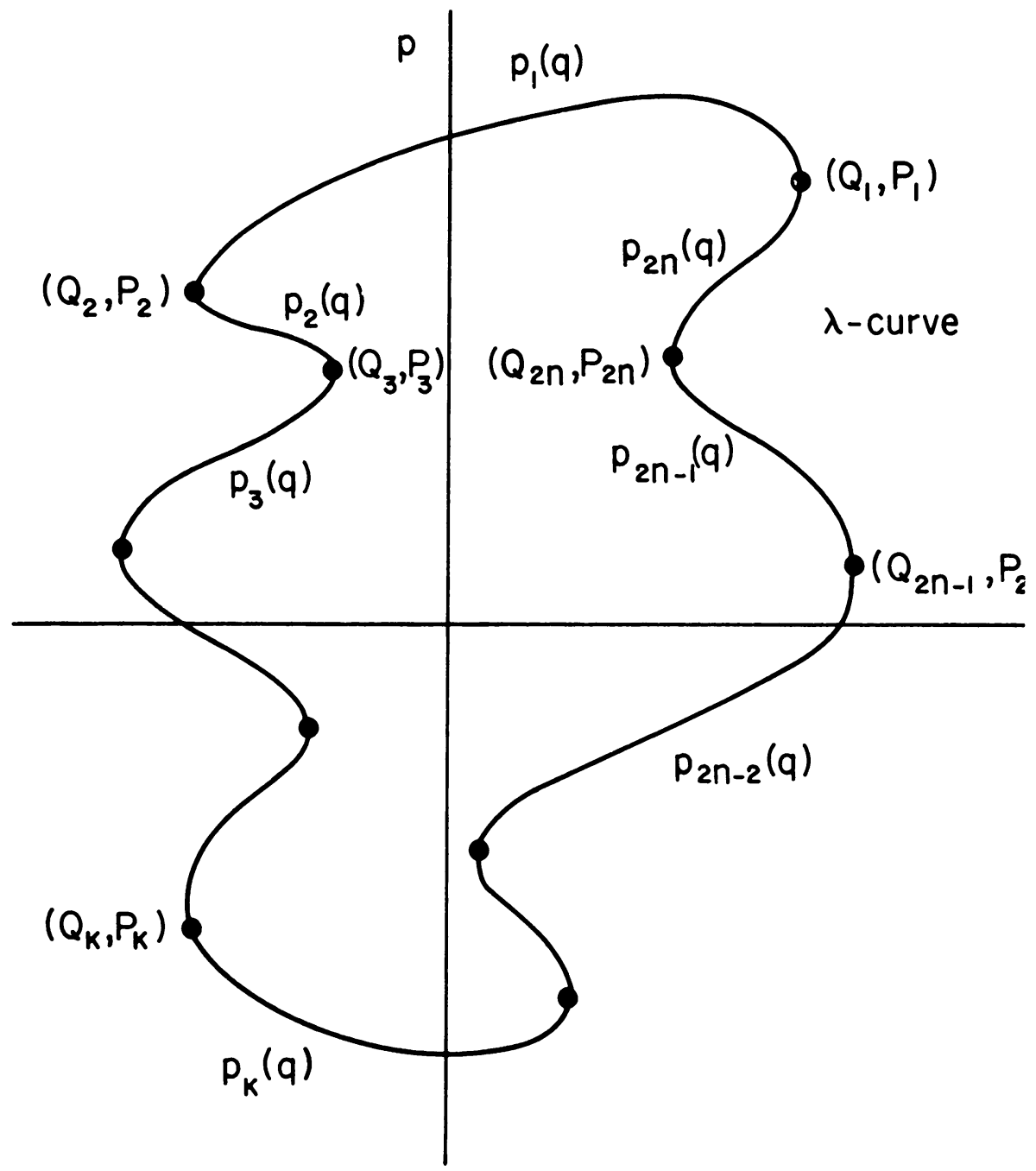

Fig. 1.

On collecting results we see that corresponding to the formal eigenvalue $\lambda$ there is the eigenfunction

$$
\psi=\frac{\exp \left[\frac{i}{\varepsilon} \int^{q} p(s) d s\right]}{\left[\tilde{K}_{p}(p(q), q)\right]^{1 / 2}}(1+O(\varepsilon)),
$$

where $p(q)$ satisfies (12). (We refer to $\psi(16)$ as an eigenfunction only in the generic sense. Further restrictions on (16) emerge as we proceed with the discussion.) The expression which appears in Eq. (16) is a generalization of that which appears in the WKB procedure $[13,14]$, and for simplicity we refer to it as a WKB solution.

A representative $\lambda$-curve is sketched in Fig. 1 . As indicated, the corresponding form of 
(12) has a number of branches, $p_{1}(q), p_{2}(q), \ldots$ Each such branch $p_{k}(q)$ exists for values of $q$ lying between the loci of vertical tangents, called turning points and denoted by $\left(P_{k}, Q_{k}\right)$ and $\left(P_{k+1}, Q_{k+1}\right)$. Outside of the interval which lies between these points, any $p_{k}$ which solves (12) must become complex, and since $\tilde{K}$ is a real form such complex branches occur in conjugate pairs.

To fix a notation we begin the indexing of turning points with one which is both left concave and at which the $\lambda$-curve is locally convex. Observe that right and left concave turning points alternate around the $\lambda$-curve (Fig. 1) and thus an even number, $2 n$, of turning points is always present. We refer back to the integral (11) and always fix the endpoint of integration as a right concave turning point and therefore a point of even index. We write

$$
\int_{Q(k)}^{q} p_{k}(s) d s
$$

with the convention that $Q(k)$ is $Q_{k}$ if $k$ is even and $Q(k)$ is $Q_{k+1}$ if $k$ is odd.

As we discuss in the next section, the form given by (16) ceases to be valid in the close neighborhood of a turning point. Moreover, for values of $q$ of which $p_{k}(q)$ lies outside its limit turning points, the expression

$$
\exp \left[\frac{i}{\varepsilon} \int_{Q(k)}^{q} p_{k}(s) d s\right]
$$

can grow exponentially large as $|q| \uparrow \infty$. Since $\psi$ must vanish at infinity such contributions must be ruled out. (This demand will determine the eigenvalue spectrum; we will see that it can be met consistently only for distinguished values of $\lambda$.) The WKB solutions must be considered separately in each of the three regions determined by the two turning points. This, however, leads to a fairly complicated formalism which we wish to avoid. To this end we agree to neglect exponentially small contributions and adopt the following convention: $p_{k}$ is always the branch of negative imaginary part for $q<Q(k)$ and of positive imaginary part to the right of the other boundary turning point. This convention guarantees that the above expression will vanish for $|q| \uparrow \infty$ and introduces errors which are at most exponentially small. Thus we regard the interval between limiting turning points as the support of a WKB solution.

Following these preliminary remarks we can express the asymptotic form of the eigenfunction by a sum,

$$
\psi_{W} \sim \sum_{k=1}^{2 n} B_{k} \psi_{W}(k)
$$

where

$$
\psi_{W}(k) \sim \frac{\exp \left[\frac{i}{\varepsilon} \int_{Q(k)}^{q} p_{k}(s) d s\right]}{\left|\tilde{K}_{p}\left(p_{k}(q), q\right)\right|^{1 / 2}}
$$

represents a WKB solution and the constants $B_{k}$ are yet to be determined. The absolute value in the denominator is permissible since $\tilde{K}_{p}$ carries a single signature on each branch. 
3. Turning point analysis. Inspection shows that the asymptotic development (17) breaks down in the neighborhood of a turning point. At such a point, (12) has a vertical tangent, and

$$
\tilde{K}_{p}(p, q)=0
$$

(As shown in the Appendix, vertical inflection points can be ignored without loss of generality.)

If we designate a typical turning point by $\left(P_{k}, Q_{k}\right)$, then the $\lambda$-curve, (12), in a neighborhood of it is approximated by the parabola

$$
0=\left(q-Q_{k}\right) \tilde{K}_{q}(k)+\frac{\left(p-P_{k}\right)^{2}}{2} \tilde{K}_{p p}(k),
$$

where for example $\tilde{K}_{q}(k)=\tilde{K}_{q}\left(P_{k}, Q_{k}\right)$. (For the present we assume that $\tilde{K}_{p p}(k) \neq 0$ - the general case is treated in the Appendix.) If we write

$$
\gamma_{k}=2 \tilde{K}_{q}(k) / \tilde{K}_{p p}(k)
$$

then the two branches in the neighborhood of a turning point are given by

$$
p=P_{k} \pm \sqrt{\gamma_{k}\left(Q_{k}-q\right)}
$$

We observe that $\gamma_{k}$ is positive when the turning point is left concave and negative when the turning point is right concave.

Condition (19) also indicates that the denominator in (18) requires expansion, and in particular

$$
\tilde{K}_{p}\left(p_{k}(q), q\right) \sim \pm \tilde{K}_{p p}(k) \sqrt{\gamma_{k}\left(Q_{k}-q\right)} .
$$

In view of the absolute value which appears in (18) the branches of (22) play no role and we write

$$
C_{k}=\left|\tilde{K}_{p p}(k) \sqrt{\gamma_{k}\left(Q_{k}-q\right)}\right|^{1 / 2}
$$

With the above expansions we can examine the WKB solution in the neighborhood of a turning point. There are four possible forms (see Fig. 2), and we first consider

$$
\psi_{W}(2 k)=\frac{\exp \left[\frac{i}{\varepsilon} \int_{Q_{2 k}}^{q} p_{2 k}(s) d s\right]}{\left|K_{p}\left(p_{2 k}(q), q\right)\right|^{1 / 2}} .
$$

For $q>Q_{2 k}$ but in the neighborhood of $Q_{2 k}$, substitution of (21) and (22) into (24) yields

$$
\psi_{W}(2 k) \sim \frac{1}{C_{2 k}} \exp \left[\frac{i}{\varepsilon} P_{2 k}\left(q-Q_{2 k}\right) \mp \frac{2 i}{3 \varepsilon} \sqrt{-\gamma_{2 k}}\left(q-Q_{2 k}\right)^{3 / 2}\right]
$$

and since $\psi_{W}(2 k-1)$ has the same left endpoint of integration but corresponds to the positive branches of (21) and (22)

$$
\psi_{W}(2 k-1) \sim \frac{1}{C_{2 k}} \exp \left[\frac{i}{\varepsilon} P_{2 k}\left(q-Q_{2 k}\right) \pm \frac{2 i}{3 \varepsilon} \sqrt{-\gamma_{2 k}}\left(q-Q_{2 k}\right)^{3 / 2}\right]
$$

The ambiguity in signature which appears in (25) and (26) rests on whether the $\lambda$-curve is locally convex in the neighborhood of $\left(P_{2 k}, Q_{2 k}\right)$. The upper branches occur when the 
$\lambda$-curve is locally convex, as in Fig. 2. The lower branch is obtained for example for the point $\left(P_{2 n}, Q_{2 n}\right)$ in Fig. 1.

To examine the WKB solutions in the neighborhood of an odd index turning point, we write

$$
\psi_{W}(2 k)=\frac{\exp \left[\frac{i}{\varepsilon} \int_{Q_{2 k}}^{Q_{2 k+1}} p_{2 k}(s) d s-\frac{i}{\varepsilon} \int_{q}^{Q_{2 k+1}} p_{2 k}(s) d s\right]}{\left|\tilde{K}_{p}\left(p_{2 k}(q), q\right)\right|^{1 / 2}}
$$

then for $q<Q_{2 k+1}$ but in the neighborhood of $Q_{2 k+1}$,

$$
\begin{aligned}
& \psi_{W}(2 k) \sim \frac{1}{C_{2 k+1}} \times \\
& \quad \exp \left[\frac{i}{\varepsilon} \int_{Q_{2 k}}^{Q_{2 k+1}} p_{2 k}(s) d s-\frac{i}{\varepsilon} P_{2 k+1}\left(Q_{2 k+1}-q\right) \mp \frac{2 i}{3 \varepsilon} \sqrt{\gamma_{2 k+1}}\left(Q_{2 k+1}-q\right)^{3 / 2}\right]
\end{aligned}
$$

Similarly,

$$
\begin{aligned}
& \psi_{W}(2 k+1) \sim \frac{1}{C_{2 k+1}} \times \\
& \exp \left[\frac{i}{\varepsilon} \int_{Q_{2 k+2}}^{Q_{2 k+1}} p_{2 k+1}(s) d s-\frac{i}{\varepsilon} P_{2 k+1}\left(Q_{2 k+1}-q\right) \pm \frac{2 i}{3 \varepsilon} \sqrt{\gamma_{2 k+1}}\left(Q_{2 k+1}-q\right)^{3 / 2}\right]
\end{aligned}
$$

The sign ambiguity occurs for the reason given above. The upper branch corresponds to Fig. 2 and the lower to the point $\left(P_{2 n-1}, Q_{2 n-1}\right)$ of Fig. 1. Beyond the range of the limit turning points each WKB solution $\psi_{W}(k)$, under the convention introduced at the close of the previous section, becomes exponentially small and therefore negligible on the scale of consideration.

Turning point analysis. The breakdown of the WKB solution (17) in the neighborhood of a turning point signals the need for a different form of the expansion of the eigenfunction in this region. Examination of any of the forms $(22,23,25,26)$ indicates that the appropriate variable in this region is

$$
\omega_{k}=\frac{Q_{k}-q}{\varepsilon^{2 / 3}}=\varepsilon^{1 / 3}\left(x_{k}-x\right)
$$

and that the appropriate form for the eigenfunction in the neighborhood of a turning point is

$$
\psi=\Psi\left(\omega_{k}\right) \exp \left[i P_{k}\left(x-x_{k}\right)\right] .
$$

In the terminology of singular perturbation theory, $\omega_{k}$ is said to be the inner variable and (31) the inner solution [15].

As preparation for the perturbation analysis in the neighborhood of a turning point we write

$$
\frac{1}{2} \varepsilon(x+y)=Q_{k}-\varepsilon^{2 / 3} \omega_{k}-\frac{\varepsilon}{2} u
$$


where as earlier $u=x-y$; then

$$
\begin{aligned}
K\left(u, \frac{1}{2} \varepsilon(x+y)\right) \sim & K\left(u, Q_{k}\right)-\varepsilon^{2 / 3} \omega_{k} K_{q}\left(u, Q_{k}\right) \\
& -\frac{1}{2} \varepsilon u K_{q}\left(u, Q_{k}\right)+\frac{\varepsilon^{4 / 3} \omega_{k}^{2}}{2} K_{q q}\left(u, Q_{k}\right) .
\end{aligned}
$$

Also

$$
\begin{aligned}
\psi(y)= & \exp \left[i P_{k}\left(y-x_{k}\right)\right] \Psi\left(\varepsilon^{1 / 3}\left(x_{k}-y\right)\right) \\
= & \left.\exp \mid-\frac{i P_{k}}{\varepsilon}\left(Q_{k}-q\right)\right] \exp \left[-i P_{k} u\right] \mid \Psi\left(\omega_{k}\right) \\
& +\varepsilon^{1 / 3} u \Psi^{\prime}\left(\omega_{k}\right)+\frac{\varepsilon^{2 / 3} u^{2}}{2} \Psi^{\prime \prime}\left(\omega_{k}\right)+\cdots \mid
\end{aligned}
$$

Then if these are substituted into the integral equation (1) we find to lowest order a form of the Airy differential equation [16]

$$
\frac{d^{2} \Psi}{d \omega_{k^{2}}}+\gamma_{k} \omega_{k} \Psi=0
$$

where $\gamma_{k}$ is defined by $(20)$.

The solution of (32) appropriate for our purposes is

$$
\Psi=A i\left(-\gamma^{1 / 3} \omega_{k}\right)
$$

(The other solution $B i\left(-\gamma^{1 / 3} \omega_{k}\right)$ must be eliminated since it grows exponentially for large positive arguments.) For large arguments (33) has the asymptotic form [16]

$$
\begin{aligned}
& A i\left(-\gamma^{1 / 3} \omega\right) \sim \frac{1}{\sqrt{\pi\left(\gamma^{1 / 3} \omega\right)^{1 / 4}}} \sin \left(\frac{\pi}{4}+\frac{2}{3}\left(\gamma^{1 / 3} \omega\right)^{3 / 2}\right), \quad \gamma^{1 / 3} \omega>0 \\
& A i\left(-\gamma^{1 / 3} \omega\right)=O\left(\exp \left[-\frac{2}{3}\left(-\gamma^{1 / 3} \omega\right)^{3 / 2}\right]\right), \quad \gamma^{1 / 3} \omega<0 .
\end{aligned}
$$

Matching procedure. It follows from these considerations that

$$
\psi(k) \propto \exp \left[-\frac{i P_{k}}{\varepsilon}\left(Q_{k}-q\right)\right] A i\left(-\gamma_{k}^{1 / 3} \omega_{k}\right) .
$$

To fix the proportionality constant we write the asymptotic form as $\left(\gamma^{1 / 3} \omega_{k} \gg 1\right)$

$$
\begin{aligned}
& \psi(k) \sim \frac{D_{k} \exp \left[-\frac{i}{\varepsilon} P_{k}\left(Q_{k}-q\right)\right]}{\left|\gamma_{k}\left(Q_{k}-q\right)\right|^{1 / 4}} \mid \exp \left[\frac{i \pi}{4}+\frac{2 i}{3 \varepsilon}\left|\gamma_{k}\right|^{1 / 2}\left|Q_{k}-q\right|^{3 / 2}\right] \\
&\left.-\exp \left[-\frac{i \pi}{4}-\frac{2 i}{3 \varepsilon}\left|\gamma_{k}\right|^{1 / 2}\left|Q_{k}-q\right|^{3 / 2}\right]\right\}
\end{aligned}
$$

where $D_{k}$ is undetermined.

Two limits are appropriate for discussion of the neighborhood of a turning point $\left(P_{k}, Q_{k}\right)$. We consider the small-argument limit (or inner limit) of (17), $\varepsilon\left|x_{k}-x\right| \ll 1$, as calculated by $(25,26,28,29)$. Since in general practice $(14)$ is referred to as the outer solution [15], this is said to be the inner limit of the outer solution. By contrast (31) is 


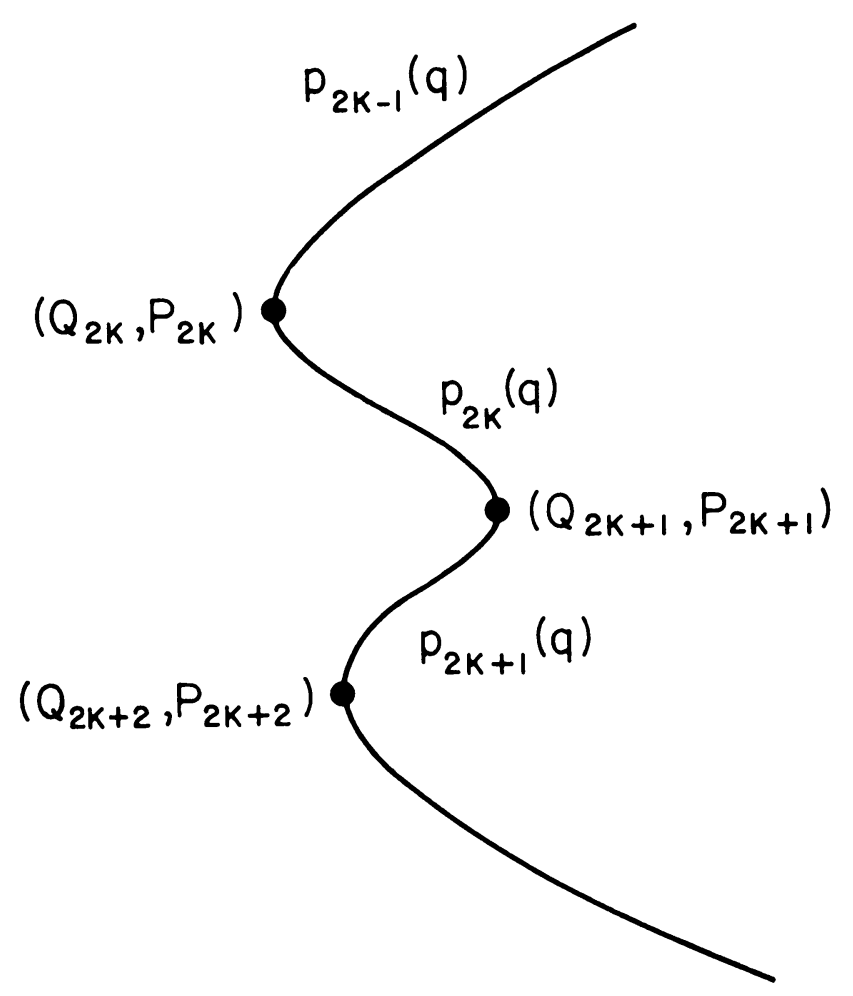

FIG. 2.

said to be the inner solution and its outer limit, $\varepsilon^{1 / 3}\left|x_{k}-x\right| \gg 1$, is given by (35). In the overlap of the two regions $\varepsilon^{1 / 3}\left|x_{k}-x\right| \gg 1$ and $\varepsilon\left|x_{k}-x\right| \ll 1$, the two forms of the solution must match.

The matching is facilitated by the following observation: in the neighborhood of any turning point only the two WKB solutions which become singular at the turning point (associated with the two branches of (21)) match to the Airy solution. We observe that contributions to (17) which are not singular at the turning point are higher-order and therefore do not enter into the matching. Secondly, although many contributions to (17) might become singular at $q=Q_{k}$, only one pair of solutions is identified with the turning point $\left(P_{k}, Q_{k}\right)$. The others carry a different sinusoidal coefficient in (31), and lead to matchings at other turning points.

We now carry out the procedure for the general case depicted in Figs. 1 and 2. Eq. (35) may be regarded as a sum of two "waves" one right-going, proportional to

$$
\exp \left[\frac{2 i}{3 \varepsilon}\left|\gamma_{k}\right|^{1 / 2}\left|Q_{k}-q\right|^{3 / 2}\right)
$$

and one left-going which is proportional to the conjugate of this expression. In (35) the ratio of coefficients of these waves is

$$
\frac{\text { coefficient of right going wave }}{\text { coefficient of left going wave }}=\exp [-i \pi / 2] \text {. }
$$

When we apply (36) we must consider the cases of odd and even indices separately. 
$\left(P_{2 k}, Q_{2 k}\right)$ : In the neighborhood of this turning point (17) becomes

$$
\psi_{W} \sim B_{2 k-1} \psi_{W}(2 k-1)+B_{2 k} \psi_{W}(2 k)
$$

which from (25) and (26) is

$$
\begin{gathered}
\psi_{W} \sim \frac{B_{2 k-1}}{C_{2 k}} \exp \left[\frac{i}{\varepsilon} P_{2 k}\left(q-Q_{2 k}\right)+\frac{2 i}{3 \varepsilon}\left|\gamma_{2 k}\right|^{1 / 2}\left(q-Q_{2 k}\right)^{3 / 2}\right] \\
+\frac{B_{2 k}}{C_{2 k}} \exp \left[\frac{i}{\varepsilon}\left(q-Q_{2 k}\right)-\frac{2 i}{3 \varepsilon}\left|\gamma_{2 k}\right|^{1 / 2}\left(q-Q_{2 k}\right)^{3 / 2}\right]
\end{gathered}
$$

In the overlap region this form, which is composed of right and left-going waves, must also satisfy (36), thus

$$
\frac{B_{2 k-1}}{B_{2 k}}=\exp [\mp i \pi / 2]
$$

where the upper sign holds if the $\lambda$-curve is convex near $\left(P_{2 k}, Q_{2 k}\right)$ and the lower if it is not.

$\left(P_{2 k+1}, Q_{2 k+1}\right)$ : We follow the above procedure and observe that in the neighborhood of this turning point

$$
\psi_{W} \sim B_{2 k} \psi_{W}(2 k)+B_{2 k+1} \psi_{W}(2 k+1)
$$

which from the introduction of (28) and (29) and the application of (36) yields

$$
\left.\frac{B_{2 k-1}}{B_{2 k+1}} \exp \left|\frac{i}{\varepsilon}\right| \int_{Q_{2 k}}^{Q_{2 k+1}} p_{2 k}(s) d s-\int_{Q_{2 k+2}}^{Q_{2 k+1}} p_{2 k+1}(s) d s \mid\right]=\exp [\mp i \pi / 2]
$$

Again the two signatures depend, respectively, on whether or not the $\lambda$-curve is locally convex at the point. It is clear from the derivation of (37) and (38) that subscripts must be taken $\bmod (2 n)$, where as before $2 n$ is the number of turning points.

If we take the appropriate form given by either (37) or (38) at each turning point and multiply these, the $B_{k}$ cancel out and we obtain

$$
\exp \left[-\frac{i}{\varepsilon} \mathscr{A}(\lambda)\right]=\exp [-i \pi]
$$

where $\mathscr{A}(\lambda)$ is the area enclosed in the $\lambda$-curve, (12). The phase at the right is $-\pi$ because a closed loop contains just two more locally convex turning points than locally concave turning points. Eq. (39) solves to give

$$
\mathscr{A}\left(\lambda_{n}\right)=(2 n+1) \pi \varepsilon
$$

where $n$ is any integer. Hence the allowable eigenvalues as they appear in (12) are restricted to the set of $\lambda$-curves which satisfy the area rule as stated in (40).

If we review the preceding arguments some simple ideas emerge. We can identify with each point of a $\lambda$-curve a phase (actually a phase difference, since a definite integral is involved). If we follow this phase once around the $\lambda$-curve we generate the enclosed area $\mathscr{A}$. On each passage through a turning point we experience a phase delay of $\pi / 2$ if it is a convex point and a phase advance of $\pi / 2$ if it is a concave point. For a proper $\lambda$-curve this results in Eq. (40), in which the term $2 n \pi$ comes from the overall indeterminancy of phase. 
Extensions to general turning points. Since area is invariant under translation and unimodular transformation, it follows that all $\widetilde{K}(p, q)$ for which the $\lambda$-curves map into each other under these transformations will have the same set of eigenvalues. Although this result was obtained under asymptotic conditions, it can be shown rigorously true [17]. The associated eigenfunctions, however, do experience a change under these transformations.

If, for example, we rotate the curve in Fig. 1, then according to the above, the operators generated in this process all have the same eigenvalues. However, it is clear that in doing this we can obtain situations not covered by our derivation of (40). In particular, the appearance of a vertical inflection point signifies a turning point at which $\widetilde{K}_{p p}=0$. Since it is an exact result that eigenvalues are invariant under rotation in the $(p, q)$-plane, a ploy for calculating eigenvalues when $\tilde{K}_{p p}=0$ at a turning point is simply to rotate the $\lambda$-curves until $\tilde{K}_{p p} \neq 0$ at all turning points. However, since it bears on the nature of the eigenfunctions, a direct derivation of the area rule (40) under these more general conditions is given in the Appendix.

4. Principal eigenvalues and eigenfunctions. As will be seen in the following, the arguments going into the area rule become suspect when the index $n$ is small. The corresponding eigenvalues and eigenfunctions are referred to as the principal eigenvalues and we now consider these in more detail. To start the discussion, we suppose that $\widetilde{K}(p, q)$ has a collection of $\lambda$-curves as depicted in Fig. 3 . The center $\left(p_{0}, q_{0}\right)$ of the closed loops is defined by

$$
\frac{\partial \tilde{K}}{\partial p}\left(p_{0}, q_{0}\right)=0=\frac{\partial \tilde{K}}{\partial q}\left(p_{0}, q_{0}\right) .
$$

It is convenient to transform the stationary point to the origin, and since this is not entirely trivial we indicate some of the steps. Define

$$
\begin{gathered}
q=q_{0}+q^{\prime}, \quad p=p_{0}+p^{\prime}, \quad x=\frac{q_{0}}{\varepsilon}+x^{\prime}, \quad y=\frac{q_{0}}{\varepsilon}+y^{\prime}, \\
\psi^{\prime}\left(x^{\prime}\right) \exp \left(-i p_{0} x^{\prime}\right)=\psi(x), \\
K^{\prime}\left(x^{\prime}-y^{\prime}, q^{\prime}\right)=K\left(x-y, q_{0}+q^{\prime}\right) \exp \left(-i p_{0}(x-y)\right),
\end{gathered}
$$

these when substituted in (1) yield

$$
\int K^{\prime}\left(x^{\prime}-y^{\prime}, \varepsilon \frac{x^{\prime}+y^{\prime}}{2}\right) \psi^{\prime}\left(y^{\prime}\right) d y^{\prime}=\lambda \psi^{\prime}\left(x^{\prime}\right) .
$$

It should be noted that $K^{\prime}$ is no longer real in general but is Hermitean, and further that the eigenfunction carries the sinusoidal factor $\exp \left(i p_{0} x^{\prime}\right)$. The Wigner transform of $\boldsymbol{K}^{\prime}$, which is real,

$$
\tilde{K}^{\prime}(p, q)=\int K^{\prime}(u, q) \exp (i p u) d u
$$

now has the required property

$$
\frac{\partial \tilde{K}^{\prime}(0,0)}{\partial q^{\prime}}=0=\frac{\partial K^{\prime}(0,0)}{\partial p^{\prime}} .
$$

Henceforth we drop the primes. 


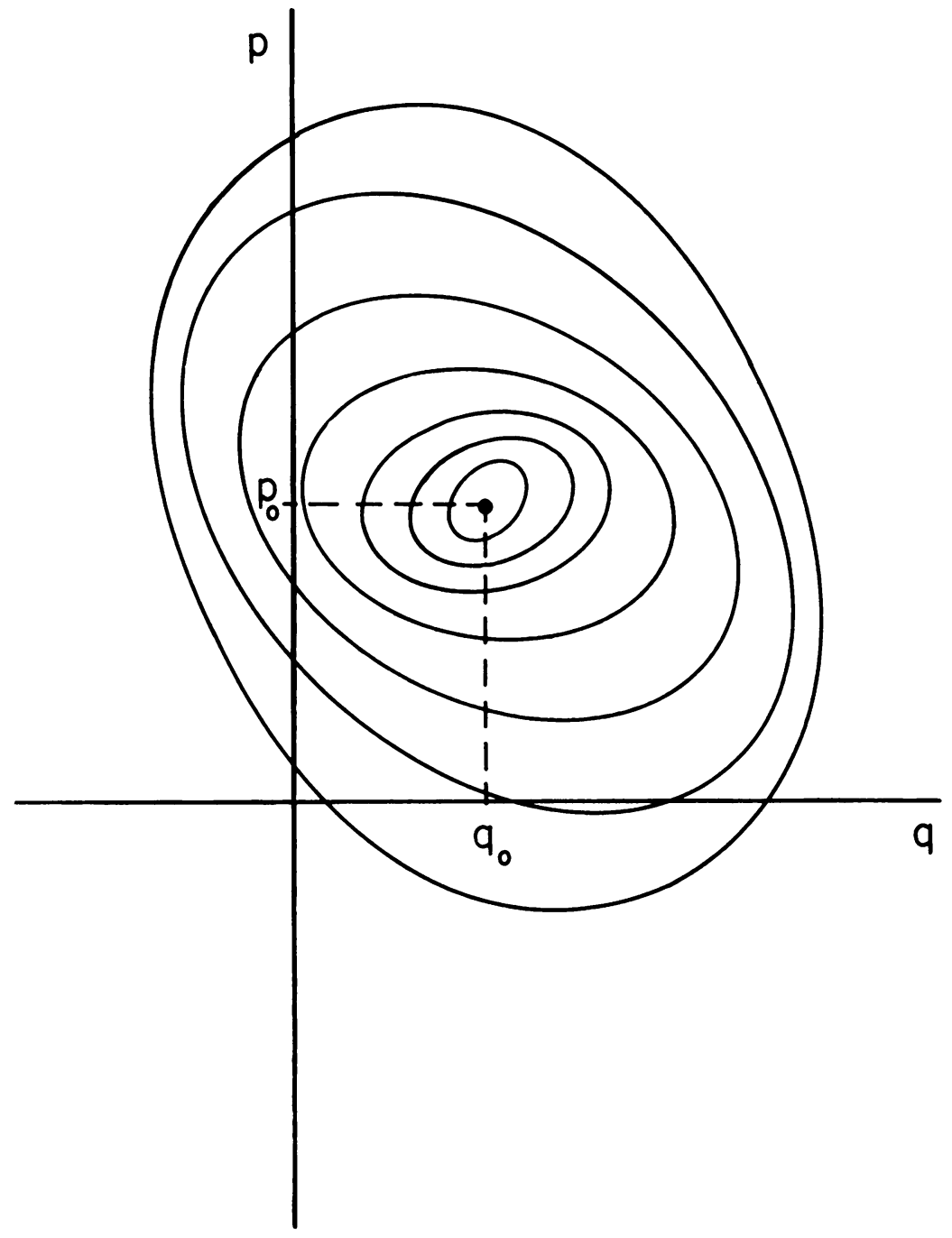

FIG. 3.

For the principal eigenvalues the area $\mathscr{A}=(2 n+1) \pi \varepsilon$ is small and we proceed with the calculation for the eigenvalues by expanding $\widetilde{K}$ in a Taylor expansion at the origin,

$$
\tilde{K}=\tilde{K}^{0}+\frac{1}{2}\left(p^{2} \tilde{K}_{p p}^{0}+2 p q \tilde{K}_{p q}^{0}+q^{2} \tilde{K}_{q q}^{0}\right)+0\left(\rho^{3}\right)=\lambda
$$

where a zero superscript indicates evaluation at the origin and $\rho^{2}=p^{2}+q^{2}$. As implied by Fig. 3, the discriminant is positive:

$$
D^{2}=\tilde{K}_{p p}^{0} \tilde{K}_{q q}^{0}-\tilde{K}_{p q}^{02}>0 .
$$

If we then calculate the area of the ellipse given implicitly by (43) we find

$$
\mathscr{A}=\frac{2\left(\lambda-\tilde{K}^{0}\right) \pi}{D} \text {. }
$$


From this the area rule yields

$$
\lambda_{n}=\tilde{K}_{0}+\frac{(2 n+1)}{2} \varepsilon D+O\left(\varepsilon^{2}\right) .
$$

This simple result is marred by the fact that the derivation of the area rule is suspect for the principal eigenvalues. To see this we observe that on the $\lambda$-curves (43) for the principal eigenvalues (45) implies that

$$
p, q=O(\sqrt{ } \varepsilon)
$$

and therefore the phase term (11) of the WKB solution has the estimate

$$
\frac{1}{\varepsilon} \int^{q} p(s) d s=O(1)
$$

Hence it is of the same order as the amplitude term, which is contrary to the assumed form of the WKB solution.

To investigate this further we can proceed from the above estimate $q=O(\sqrt{ } \varepsilon)$. From this it follows that the eigenfunctions corresponding to the principal eigenvalues can be written

$$
\psi=\psi(\sqrt{ } \varepsilon x)
$$

and hence (1) becomes

$$
\int K\left(x-y, \varepsilon\left(\frac{x+y}{2}\right)\right) \psi(\sqrt{ } \varepsilon y) d y=\lambda \psi(\sqrt{ } \varepsilon x) .
$$

To treat this case it is convenient to introduce new variables

$$
\delta=\sqrt{\varepsilon}, \quad r=\delta x, \quad s=\delta y
$$

which yields

$$
\int \frac{1}{\delta} K\left(\frac{r-s}{\delta}, \delta\left(\frac{r+s}{2}\right)\right) \psi(s) d s=\lambda \psi(r) .
$$

The kernel $K$ is a peaking function in its first argument, which in turn suggests the following expansions;

$$
\begin{aligned}
\psi(s)=\psi(r)-\delta\left(\frac{r-s}{\delta}\right) \psi^{\prime}(r)+\frac{\delta^{2}}{2}\left(\frac{r-s}{\delta}\right)^{2} \psi^{\prime \prime}(r)+\cdots & , \\
K\left(\frac{r-s}{\delta}, \frac{\delta}{2}(r+s)\right)=K^{0}\left(\frac{r-s}{\delta}\right)+\delta r K_{q}^{0}\left(\frac{r-s}{\delta}\right) & -\delta^{2}\left(\frac{r-s}{\delta}\right) K_{q}^{0}\left(\frac{r-s}{\delta}\right) \\
& +\frac{r^{2}}{2} \delta^{2} K_{q q}^{0}\left(\frac{r-s}{\delta}\right)+O\left(\delta^{3}\right),
\end{aligned}
$$

where a zero superscript indicates that the second argument is set to zero. Substitution of these into (47) then yields the second-order Hermitean operator equation

$$
-\tilde{K}_{p p}^{0} \frac{d^{2}}{d r^{2}} \psi+r^{2} \tilde{K}_{q q}^{0} \psi-i \tilde{K}_{p q}^{0}\left(r \frac{d}{d r}+\frac{d}{d r} r\right) \psi=\Lambda \psi,
$$


with

$$
\Lambda=2\left(\lambda-\widehat{K}^{0}\right) / \delta^{2}
$$

In deriving (50) we have used (42).

The eigenfunctions of (50) are given by [17]

$$
\psi=\exp \left(-\frac{i r^{2}}{2} \frac{\tilde{K}_{p q}^{0}}{K_{p p}}\right) h_{n}\left(r \sqrt{\frac{D}{\tilde{K}_{p p}^{0}}}\right)=\exp \left(-\frac{i \varepsilon x^{2}}{2} \frac{\tilde{K}_{p q}^{0}}{\tilde{K}_{p p}^{0}}\right) h_{n}\left(x \sqrt{\frac{\varepsilon D}{\tilde{K}_{p p}^{0}}}\right)
$$

where $h_{n}(x)$ is the Hermite function (see (58)). The corresponding eigenvalues are

$$
\Lambda=(2 n+1)
$$

which on substitution into (51) lead to same result for $\lambda_{n}$ as given by the area rule, namely (45).

Thus the area rule remains valid even for the principal eigenvalues provided $D(44)$ is bounded away from zero. The form of the eigenfunctions, however, is modified and is now given by (52) (see also (41)) instead of the WKB form. In [18] various additional examples are discussed, in particular cases for which $D$ vanishes or does not exist. In such cases the above results must be modified.

5. Special cases. The purpose of the present section is to make contact with two known cases which fall within the framework of our results. An extended and diverse class of cases are presented in [18].

Ordinary differential equations. In the classical theory of ordinary differential equations one encounters the eigenvalue problem

$$
\frac{d^{2}}{d x^{2}} \psi+\{\lambda-r(x)\} \psi(x)=0, \quad-\infty<x<\infty
$$

for $\psi$ square-integrable [19]. Then for smooth convex $r$ such that $r(x) \uparrow \infty,|x| \uparrow \infty$ sufficiently rapidly, one may show [19]

$$
\int_{X_{n}^{\prime}}^{X_{n}}\left\{\lambda_{n}-r(x)\right\}^{1 / 2} d x=\pi\left(n+\frac{1}{2}\right)+O\left(\frac{1}{n}\right)
$$

where $r\left(X_{n}\right)=r\left(X_{n}^{\prime}\right)=\lambda_{n}$.

To relate this to the area rule calculation, consider the kernel

$$
K\{x, y\}=r\left(\frac{x+y}{2}\right) \delta(x-y)-\delta^{\prime \prime}(x-y)
$$

where $\delta$ represents the delta function and $\delta^{\prime \prime}$ its second derivative. It is clear that

$$
\int_{-\infty}^{\infty} K\{x, y\} \psi(y) d y=\lambda \psi(x)
$$

is a restatement of (53). If we leave aside for the moment the question of a small parameter and proceed with the area rule calculation, then we must first compute $\tilde{K}$ which in this case is

$$
\tilde{K}=p^{2}+r(q)
$$


whence $p(q)= \pm\left\{\lambda_{n}-r(q)\right\}^{1 / 2}$ on the contour line $\widetilde{R}(p, q)=\lambda_{n}$. To see the equivalence of (54) with the area rule (40) (for $\varepsilon=1$ ), we simply note that the left-hand side of (54) is exactly one-half the area enclosed in the $\lambda$-curve.

Unlike our development of area rule which required a slowness scale $\varepsilon$, the above treatment is derived solely under the assumption that $n \uparrow \infty$. To see the connection, observe first that $\lambda_{n} \uparrow \infty$ under this limit and second that for $\lambda$ large the scale of variation in (53) is $1 / \sqrt{ } \lambda$. Thus let $\lambda_{0}$ be typical of the size of $\lambda$ and set $x \sqrt{ } \lambda_{0}=x^{\prime}$; then (53) becomes

$$
\frac{d^{2} \psi}{d x^{\prime 2}}-\left[\frac{\lambda}{\lambda_{0}}-\frac{1}{\lambda_{0}} r\left(\frac{1}{\sqrt{\lambda_{0}}} x\right)\right] \psi=0
$$

and $1 / \sqrt{ } \lambda_{0}$ is seen to act as the $\varepsilon$ for the problem. Alternately, if a slowness scale is present, e.g.

$$
\frac{d^{2} \psi}{d x^{2}}-[\lambda-r(\varepsilon x)] \psi=0,
$$

then the variable change $\varepsilon x=x^{\prime}$ gives

$$
\frac{d^{2}}{d x^{\prime 2}} \psi-\left[\frac{\lambda}{\varepsilon^{2}}-\frac{1}{\varepsilon^{2}} r\left(x^{\prime}\right)\right] \psi=0
$$

which implies a large "eigenvalue" $\lambda / \varepsilon^{2}$.

A solvable kernel. It will be illuminating to compare our asymptotic results with a specific exact calculation. We begin with a formula due to Mehler [20]:

$$
\begin{aligned}
K\{x, y\} & =\frac{1}{\left(1-z^{2}\right)^{1 / 2}} \exp \left[-\left(\frac{x-y}{2}\right)^{2}\left(\frac{1+z}{1-z}\right)-\left(\frac{x+y}{2}\right)^{2}\left(\frac{1-z}{1+z}\right)\right] \\
& =\sum_{n=0}^{\infty} \frac{h_{n}(x) h_{n}(y)}{2^{n} n !} z^{n}
\end{aligned}
$$

where $h_{n}$ denotes the Hermite functions [20]

$$
h_{n}(x)=H_{n}(x) \exp \left(-x^{2} / 2\right) \text {. }
$$

$H_{n}(x)$ is the Hermite polynomial [20] of degree $n$ defined by the generating function

$$
F(u, x)=\exp \left(2 u x-u^{2}\right)=\sum_{n=0}^{\infty} u^{n} H_{n}(x) / n !
$$

Then since

$$
\int_{-\infty}^{\infty} h_{n}(x) h_{m}(x) d x=\delta_{n m} \sqrt{ } \pi 2^{n} n !
$$

we have

$$
\int_{-\infty}^{\infty} K\{x, y\} h_{n}(y) d y=\Lambda_{n} h_{n}(x)
$$

with

$$
\Lambda_{n}=\sqrt{ } \pi z^{n}
$$


By a substitution of variables the role of a slow dependence in (57) may be brought out clearly. It is perfectly general that if the Hermitean kernel $K(x, y)$ has a given spectrum then the rescaled kernel

$$
S\{x, y\}=\alpha K\{\alpha x, \alpha y\}
$$

has the same spectrum (with eigenfunctions $\psi_{n}(\alpha x)$ ). If we substitute (57) in (61) and let

$$
\frac{1}{\alpha}=\left(\frac{1-z}{1+z}\right)^{1 / 2}=\beta^{1 / 2}
$$

we see that

$$
S\{x, y\}=\frac{1+\beta}{2} \exp \left[-\left(\frac{x-y}{2}\right)^{2}\right] \exp \left[-\left(\beta \frac{x+y}{2}\right)^{2}\right] .
$$

Evidently small $\beta$ yields a slow dependence in the $x+y$ variable and plays the role of $\varepsilon$ in our development of the area rule. In terms of the $\beta$ variable, the exact eigenvalues (60) become

$$
\Lambda_{n}=\sqrt{ } \pi[(1-\beta) /(1+\beta)]^{n}
$$

If we set $q=\beta(x+y) / 2$ and transform we find

$$
\tilde{S}(p, q)=\sqrt{ } \pi(1+\beta) \exp \left[-\left(p^{2}+q^{2}\right)\right],
$$

and since $\mathscr{A}=\left(p^{2}+q^{2}\right) / \pi$, the area rule $(40)$ yields

$$
\lambda_{n}=\sqrt{ } \pi(1+\beta) \exp [-(2 n+1) \beta] .
$$

To compare this with the exact value (64), we write the latter as follows:

$$
\begin{aligned}
\Lambda_{n} & =\sqrt{ } \pi(1+\beta) \exp [n \ln (1-\beta)-(n+1) \ln (1+\beta)] \\
& =\sqrt{ } \pi(1+\beta) \exp \left[-(2 n+1) \beta+\beta^{2}+O\left(n \beta^{3}\right)\right]
\end{aligned}
$$

and hence

$$
\Lambda_{n} / \lambda_{n}=\exp \left[\beta^{2}+O\left(n \beta^{3}\right)\right] .
$$

It is evident that $\lambda_{n}$ and $\Lambda_{n}$ agree through linear terms in $\beta$ at least up until $n=o\left(1 / \beta^{2}\right)$.

To understand the reason for breakdown of area rule at large values of the index $n$ we must return to the WKB solution. In particular, we note that for the example at hand the denominator of $(18)$ is

$$
\sqrt{\tilde{K}_{p}}=\sqrt{-2 p \lambda_{n}} .
$$

Since the product contained under the radical is easily seen to vanish for $n \uparrow \infty$, the derivation itself signals the breakdown for large index. It is important to note that the breakdown is not generic but particular to the case at hand. The area rule for the ordinary differential equation considered above, for example, remains valid as $n \uparrow \infty$. In any particular case one must reexamine the WKB solution for self-consistency in order to appraise the validity of the area rule for large index.

The nonuniformity (67) asserts itself only for those eigenfunctions which are least important in a practical sense. Thus if, for example, we use (66) to estimate the trace of 
$S\{x, y\}$, we find

$$
\sum_{n=0}^{\infty} \lambda_{n}=\sqrt{ } \pi \frac{1+\beta}{2 \sinh \beta} \sim \sqrt{ } \pi \frac{1+\beta}{2 \beta}=\sum_{n=0}^{\infty} \Lambda_{n}
$$

This underlines the fact that the estimate (66) only becomes poor when $n \uparrow \infty$, in which case $\lambda_{n} \rightarrow 0$.

Finally, we remark that the area rule gives a respectable estimate even when $\beta$ is not small. Even if we choose $\beta=1$, which gives the product kernel

$$
S(x, y)=\exp \left[-\frac{x^{2}}{2}\right\rfloor \exp \left[-\frac{y^{2}}{2}\right)
$$

and only one non-zero eigenvalue (and a $x+y$ dependence which is hardly slow), we find $\lambda_{0} / \Lambda_{0}=2 / e=.7358$; the area rule misses by only $27 \%$.

Appendix. In this appendix we consider the derivation of the area rule (40) when higher-order turning points are present.

If we write

$$
\left(d^{n} / d p^{n}\right) \widehat{K}=\widetilde{K}_{p}^{(n)},
$$

then the $\lambda$-curve in the neighborhood of any turning point $\left(P_{k}, Q_{k}\right)$ is given by

$$
0=\tilde{K}_{q}(k)\left(q-Q_{k}\right)+\frac{\left(p-P_{k}\right)^{n}}{n !} \tilde{K}_{p}^{(n)}(k), \quad n \geq 2 .
$$

The case when $n=2$ was considered above. A discussion similar to that leading to $(25,26,28,29)$ indicates a dependence on the variable

$$
\frac{n}{n+1}\left|\frac{n ! \tilde{K}_{q}(k)}{K_{p}^{(n)}(k)}\right|\left|q-Q_{k}\right|^{1+1 / n}
$$

which for $n=2$ is that found in those expressions. This in turn suggests

$$
\omega_{k}=\varepsilon^{1 /(n+1)}\left(x_{k}-x\right)
$$

as an inner variable (as in (30)). The inner form of an eigenfunction is again (31), but in general $\psi$ satisfies

$$
\frac{d^{n}}{d \omega_{k}^{n}} \Psi-\left(\frac{n !(-i)^{n} \tilde{K}_{q}(k)}{\tilde{K}_{p}^{n}(k)}\right) \omega_{k} \Psi=0 .
$$

Eq. (A.2) has an irregular singular point at $\infty$ and has just one solution which vanishes for $\omega_{k} \rightarrow \pm \infty$. This solution is easily obtained by transforms and is

$$
\Psi=\int_{-\infty}^{\infty} \exp \left[-i\left\{\omega_{k} \xi-\frac{\xi^{n+1}}{n+1}\left(\frac{\tilde{K}_{p}^{(n)}(k)}{n ! \widetilde{K}_{q}(k)}\right)\right\}\right] d \xi .
$$

For the case $n=2$ we recover the Airy function considered above, (33).

As in the case already treated, the matching procedure requires expansion of $\Psi$ (A.3) for large values of the argument $\left|\omega_{k}\right|$. For these asymptotics a variety of situations obtain, depending on the parity of $n$, the signature of $\omega_{k}$, and the signature of

$$
\phi=\tilde{K}_{p}^{(n)}(k) / n ! \tilde{K}_{q}(k) \text {. }
$$


In any case one must examine (A.3) for the stationary values of the exponent, when

$$
\frac{d}{d \xi}\left(\xi \omega_{k}-\frac{\xi^{n+1}}{n+1} \phi\right)=\omega_{k}-\xi^{n} \phi=0
$$

or for

$$
\xi=\xi_{0}=\left(\frac{\omega_{k}}{\phi}\right)^{1 / n}, \quad \phi=\tilde{K}_{p}^{(n)}(k) / n ! \tilde{K}_{q}(k) .
$$

Further discussion is conveniently divided into two cases depending on the parity of $n$.

$n$ even: For $\omega_{k} / \phi \ll 0$ (A.3) can be evaluated by a saddle-point analysis [14]. The result is that

$$
\Psi=O\left(\exp \left[-\frac{n}{n+1} \frac{\left|\omega_{k}\right|^{(n+1) / n}}{|\phi|^{1 / n}} \sin \frac{\pi}{n}\right]\right) .
$$

This generalizes the estimate given after (34) and corresponds to $\omega_{k}$ lying beyond the turning points when the branch $p(q)$ is complex.

For $\omega_{k} / \phi \gg 0,(4.3)$ can be asymptotically evaluated by Kelvin's formula [14] and the result is

$$
\Psi \sim 2\left(\frac{2 \pi}{\left|\phi \xi_{0}^{n-1}\right|}\right)^{1 / 2} \sin \left(\frac{n}{n+1}\left(\frac{\omega_{k}}{\phi^{1 /(n+1)}}\right)^{(n+1) / n}+\frac{\pi}{4}\right) .
$$

Aside from an unimportant multiplicative constant, when $n=2$ this is the same as (34).

The main feature to note in (A.5) is the phase factor $\pi / 4$. An examination of the matching procedure given earlier shows that the ultimate derivation of (40) rests simply on the presence of $\pi / 4$ in (34). In fact we can also regard (A.5) as composed of right- and left-going "waves", and are then again led to Eq. (36). Since the matching procedure depends on just this property it again goes through as before with the area rule (40) as a result.

$n$ odd: The situation is now somewhat different. On the geometric side we are speaking of a vertical inflection point of third or higher order, with just one branch either side of it. On the analytical side we observe from (A.4) that the stationary point $\xi_{0}$ is now on the real line independently of the signature $\omega_{k} / \phi$. If we again appeal to Kelvin's formula for the asymptotic evaluation of (A.3), the result is

$$
\Psi \sim\left(\frac{2 \pi}{\left|\phi \xi_{0}^{n-1}\right|}\right)^{1 / 2} \exp \left[-i\left|\frac{n}{\mid n+1} \omega_{k}\left(\frac{\omega_{k}}{\phi}\right)^{1 / n}-\frac{\pi}{4} \operatorname{sgn} \phi\right|\right] .
$$

In this case we have in effect an incident and a transmitted "wave", and (A.6) states that no phase change takes place at this type of turning point. Referring back to the WKB solution (17), this states that the two coefficients corresponding to the two branches on either side of an inflection point are the same. Thus, in effect, inflection turning points can be ignored for purposes of evaluating the coefficients $B_{k}$, and the analysis reduces to the cases which have already been considered.

\section{REFERENCES}

[1] F. Ratliff, Mach bands: quantitative studies on neural networks in the retina, Holden-Day, San Francisco, 1967

[2] T. N. Cornsweet, Visual perception, Academic Press, New York, 1970 
[3] H. R. Wilson and S. C. Giese, Threshold visibility of frequency gradient patterns, Vision Res. 17, 1177-1190 (1977)

[4] J. O. Limb and C. B. Rubenstein, A model of threshold vision incorporating inhomogeneity of the visual field, Vision Res. 17, 571-584 (1977)

[5] A. J. Doorn, J. J. Koendrink, and M. A. Bouman, The influence of the retinal inhomogeneity on the perception of spatial patterns, Kybernetic 10, 223-230 (1972)

[6] J. E. Robson and N. Graham, Probability summation and regional variation in sensitivity across the visual field, Vision Res. (in press)

[7] L. Sirovich, Boundary effects in neutral networks, SIAM J. Appl. Math., 39, 142-160 (1980)

[8] L. Sirovich, S. Brodie, and B. W. Knight, The effect of boundaries on the response of a neural network, Biophys. J. 28, 423-446 (1979)

[9] E. P. Wigner, On the quantum correction for thermodynamic equilibrium, Phys. Rev. 40, 749-759 (1932)

[10] H. J. Groenewald, On the principles of quantum mechanics, Physica 12, $405-460$ (1946)

[11] J. E. Moyal, Quantum mechanics as a statistical theory, Proc. Camb. Phil. Soc. 45, 99-124 (1949)

[12] J. Bruer, The classical limit of quantum theory, Ph.D. thesis, The Rockefeller University, 1978

[13] J. Heading, An introduction to phase-integral methods, Methuen \& Co. Ltd., London, 1962

[14] L. Sirovich, Techniques of asymptotic analysis, Springer-Verlag, New York, 1971

[15] J. Cole, Perturbation techniques in applied mathematics, Blaisdell, Waltham, Mass. 1968

[16] M. Abramowitz and I. A. Stegun, Handbook of mathematical functions, U.S. Government Printing Office,

[17] B. W. Knight and L. Sirovich, The Wigner transform and some exact properties of linear operators, submitted for publication

[18] L. Sirovich and B. W. Knight, Contributions to the eigenvalue problem for slowly varying operators, submitted for publication

[19] E. C. Titchmarsh, Eigenfunction expansions associated with second-order differential equations, pt. 1, 2nd ed., Oxford University Press, London, 1962

[20] A. Erdelyi, ed., Bateman manuscript project, higher transcendental functions, vol. 2, p. 194, McGraw-Hill, New York, 1953 